\title{
Target-absent controls in blocking experiments with rats
}

\author{
KATHLEEN M. TAYLOR \\ Barnard College, New York, New York \\ VICTORY T. JOSEPH \\ Columbia University, New York, New York \\ Peter D Balsam \\ Barnard College, New York, New York \\ and Columbia University, New York, New York \\ AND \\ M. E. BitTERman \\ University of Hawaii, Honolulu, Hawaii
}

\begin{abstract}
In three between-groups blocking experiments with rats, two concurrent and one forward, several common control procedures were employed: Reinforced trials with the putative blocking stimulus were either omitted entirely (Kamin control), replaced by unsignaled reinforcements (Wagner control), or replaced by reinforced trials with a different stimulus $(\mathrm{C}+$ control). In each experiment, parallel treatments with the target stimulus absent during training served to examine the possibility that differential responding in tests with the target stimulus might be traced solely to differential exposure to the nontarget stimuli. In Experiment 1, responding by a concurrent blocking group during the test was no different than responding by a Kamin control group, and responding by a Wagner control group was greater than that of either of the other groups - a pattern of results, mirrored in the performance of the target-absent groups, that could be attributed to the elevation of contextual excitation by unsignaled reinforcement. In Experiment 2, responding in the test by a concurrent blocking group was no different than that by a $\mathrm{C}+$ control group. In Experiment 3, a finding of less responding by a forward blocking group than by a $\mathrm{C}+$ control group when the target stimulus was present during training, but not when it was absent, provided plausible evidence of blocking.
\end{abstract}

In the prototypical blocking experiment (Kamin, 1969), reinforced trials with stimulus $\mathrm{A}(\mathrm{A}+$ trials) given before reinforced trials with a compound of the stimuli $\mathrm{A}$ and $\mathrm{B}$ $(\mathrm{AB}+$ trials) were found to reduce subsequent responding to $\mathrm{B}$ alone, relative to a control group for which the A+ trials were simply omitted in training. Subsequent responding to $\mathrm{B}$ alone was reported to also be reduced by $\mathrm{A}+$ trials that did not precede the $\mathrm{AB}+$ trials, but were instead interspersed among them (Wagner, 1969), an effect that has been described as "concurrent" blocking, to distinguish it from Kamin's "forward" blocking. Various other control procedures have been used in efforts to establish that the conditioning of A is essential to these effects, rather than the additional experience with either reinforcement apart from A or A apart from reinforcement. In his work on "cue validity," Wagner substituted unsignaled reinforcements for the A+ trials, thus controlling for experience with reinforcement but leaving experience with A unequated. In later experiments, A was either backwardly paired or explicitly unpaired with reinforcement (thus equating experience both with $\mathrm{A}$ and with reinforcement), and reinforced trials with a different stimulus, $\mathrm{C}+$, were substituted for the $\mathrm{A}+$ trials; in experiments with honeybees, Smith and Cobey (1994) notably used all three of these control procedures, in addition to Kamin's. Although none of these controls is entirely satisfactory from a purely formal point of view, the same outcome has generally been reported for all of them - that is, less responding by the blocking animals than by the control animals in tests with $\mathrm{B}$. The traditional explanation has been that the conditioning of $\mathrm{B}$ on $\mathrm{AB}+$ trials is impaired by the conditioning of $\mathrm{A}$, with which $\mathrm{B}$ competes for either associative strength (Rescorla \& Wagner, 1972) or attention (Sutherland \& Mackintosh, 1971). A later idea (Miller \& Matzel, 1988) was that blocking is a performance effect rather than a learning effect - that is, the pairing of A and B establishes a relation between them such that responding to each alone is an increasing function of its associative strength relative to the strength of the other.

Prompted, as they explained, by doubts as to the adequacy of the various conventional control procedures, Blaser, Couvillon, and Bitterman (2006) recently repeated, with only one variation, some earlier experiments (Blaser, Couvillon,

M. E. Bitterman, jeffb@pbrc.hawaii.edu 
\& Bitterman, 2004) that had given the appearance of blocking in honeybees. The new experiments differed from the earlier ones in that the target stimulus (B) was absent during training and was encountered for the first time in the tests, but even so, less responding to the target stimulus occurred in the blocking groups than in the control groups. Consider, for example, an experiment in which less responding to $\mathrm{B}$ (the target stimulus) was found after $8 \mathrm{~A}+$ trials (reinforced trials with the putative blocking stimulus) followed by 8 $\mathrm{AB}+$ trials (reinforced trials with the $\mathrm{AB}$ compound) than after 8 unsignaled reinforcements followed by $8 \mathrm{AB}+$ trials (the Wagner control): Similar results were obtained in a target-absent version of the same experiment-that is, less responding in the test with $\mathrm{B}$ after $16 \mathrm{~A}+$ trials than after 8 unsignaled reinforcements followed by $8 \mathrm{~A}+$ trials. As Rescorla (1981) warned decades ago (to little effect) and the new honeybee results demonstrate, differential experience with nontarget stimuli in conventional between-groups blocking experiments may, at least in some cases, produce differential responding in tests with the target stimulus independently of prior experience with it - independently, that is, of opportunity to learn about it. A demonstration of blocking in such an experiment therefore can carry little conviction unless accompanied by null results for parallel groups trained with the target absent.

In the present experiments with rats, which were designed to look for evidence of blocking demonstrably dependent on experience with B, we used both the Kamin and the Wagner controls, which were chosen largely for historical reasons, and also the $\mathrm{C}+$ control, intuitively perhaps the most satisfactory of the various controls, although it is certainly open to some question (Blaser et al., 2006, p. 69). Because of its greater operational convenience, we began with concurrent training, but we also used the forward procedure, both to make contact with the main body of blocking literature and

Table 1

Experimental Designs and Results

\begin{tabular}{|c|c|c|c|}
\hline Group & Test B & Test A & Test C \\
\hline \multicolumn{4}{|c|}{ Experiment 1: Concurrent Kamin-Wagner } \\
\hline 1. $\mathrm{A}+/ \mathrm{AB}+$ & $12(5)$ & $29(3)$ & $1(1)$ \\
\hline 2. $-/ \mathrm{AB}+$ & $15(1)$ & $19(1)$ & $2(1)$ \\
\hline 3. $+/ \mathrm{AB}+$ & $23(17)$ & $20(6)$ & $4(4)$ \\
\hline 4. $\mathrm{A}+/ \mathrm{A}+$ & $4(9)$ & $45(2)$ & $2(1)$ \\
\hline 5. $-/ \mathrm{A}+$ & $1(3)$ & $36(0)$ & $0(0)$ \\
\hline 6. $+/ \mathrm{A}+$ & $8(17)$ & $42(7)$ & $3(2)$ \\
\hline \multicolumn{4}{|c|}{ Experiment 2: Concurrent $\mathrm{C}+$} \\
\hline 1. $\mathrm{A}+/ \mathrm{AB}+$ & $12(7)$ & $37(3)$ & $9(3)$ \\
\hline 2. $\mathrm{C}+/ \mathrm{AB}+$ & $14(3)$ & $21(3)$ & $24(2)$ \\
\hline 3. $\mathrm{A}+/ \mathrm{A}+$ & $5(5)$ & $39(3)$ & $3(3)$ \\
\hline 4. $\mathrm{C}+/ \mathrm{A}+$ & $5(7)$ & $22(5)$ & $20(2)$ \\
\hline \multicolumn{4}{|c|}{ Experiment 3: Forward $\mathrm{C}+$} \\
\hline 1. $\mathrm{A}+\rightarrow \mathrm{AB}+$ & $5(1)$ & $26(1)$ & $8(0)$ \\
\hline 2. $\mathrm{C}+\rightarrow \mathrm{AB}+$ & $14(5)$ & $18(0)$ & $29(1)$ \\
\hline 3. A+ $\rightarrow \mathrm{A}+$ & $2(1)$ & $32(1)$ & $8(1)$ \\
\hline 4. $\mathrm{C}+\rightarrow \mathrm{A}+$ & $3(3)$ & $28(2)$ & $30(2)$ \\
\hline
\end{tabular}

Note-A, B, and C denote the stimuli; + , reinforcement; - , blank trial; /, concurrent training; $\rightarrow$, sequential training. The mean number of responses in all 10 test trials with each of the three stimuli is shown, along with (in parentheses) the mean number of responses in the corresponding pre-CS intervals. because, as Wagner (1969) early suggested, the forward procedure might be expected, at least from some viewpoints, to be more effective than the concurrent procedure.

The designs of our experiments are summarized in Table 1. In Experiment 1 (concurrent Kamin-Wagner), there were six groups of subjects: (1) a blocking group; (2) a Kamin control group, for which the A+ trials were simply omitted; (3) a Wagner control group, for which A+ trials were replaced by unsignaled reinforcements; and (4-6) three parallel B-absent groups. In Experiment 2 (concurrent $\mathrm{C}+$ ), we included (1) a blocking group; (2) a C+ control group, for which the $\mathrm{A}+$ trials were replaced by reinforced trials with a different stimulus; and (3-4) two parallel B-absent groups. Experiment 3 (forward $\mathrm{C}+$ ) was like Experiment 2 in all respects, except that the training was sequential rather than concurrent, so that $\mathrm{A}+$ or $\mathrm{C}+$ trials in the first stage of training were followed by $\mathrm{AB}+$ or $\mathrm{A}+$ trials in the second.

\section{METHOD}

The subjects were 350 - to 400 -g male CRL:CD rats (Charles River Laboratories, Kingston, NY) living in a controlled laboratory environment (12:12-h light:dark cycle), to which they were adapted for several weeks before experimental work with them was begun. They were maintained on a 23-h schedule of food deprivation, with water delivered ad lib in the home cages. The training was done in a set of commercial operant chambers (Model ENV-008; MED Associates, St. Albans, VT) contained in sound-attenuating cabinets. Each was equipped with several light sources, a speaker, and a food trough into which reinforcement consisting of two $45 \mathrm{mg}$ of food pellets (BioServ, Frenchtown, $\mathrm{NJ}$ ) was dropped by a pellet dispenser; entries of an animal's head into the trough were detected by a photocell in its rim. Prior to the beginning of each experiment, three sessions of feeder training were presented, during each of which - under only the dim, constant illumination provided by a small red signal lamp - the animals learned to take pellets dropped into the trough on a variable-time 45 -sec schedule.

In Experiment 1 (concurrent Kamin-Wagner), six groups of 8 animals each (see Table 1) were trained in 20 daily sessions. The target stimulus (B) was a white signal light above and to the left of the food trough. The putative blocker (A) was an auditory stimulus, an 80-dB white noise for half of the animals in each group and an $80-\mathrm{dB}, 1000-\mathrm{Hz}$ tone for the rest. These stimuli were later used in balanced roles as $\mathrm{A}$ and $\mathrm{C}$, in the two subsequent experiments with the $\mathrm{C}+$ control procedure. For Group 1, in each training session, $10 \mathrm{~A}+$ trials and $10 \mathrm{AB}+$ trials were scheduled in quasirandom sequence on a variable-interval schedule that averaged $180 \mathrm{sec}$; the interval between the onset of the conditioned stimulus (CS) and the reinforcement was $12 \mathrm{sec}$. The A+ trials were replaced with blank trials for Group 2, and with the pellets alone for Group 3. The procedures for Groups 4-6 were exactly the same as those for Groups 1-3, respectively, except that the target stimulus was never presented. After the training sessions, 3 test sessions were held on successive days, with B presented on the first day, A on the second, and C (the auditory stimulus not previously encountered) on the third. Each test session included 10 
trials at variable intervals that averaged $180 \mathrm{sec}$, on each of which the stimulus to be tested was presented without reinforcement for $12 \mathrm{sec}$. Head entries into the food trough were recorded both during the CS and in the immediately preceding interval of $12 \mathrm{sec}$ (the pre-CS interval).

In Experiment 2 (concurrent $\mathrm{C}+$ ), four groups of 12 subjects were each trained in 20 sessions (see Table 1). The training procedure was the same as in Experiment 1, except that $15 \mathrm{~A}+$ and $5 \mathrm{AB}+$ trials (rather than 10 trials of each) were scheduled in each training session for the first group, and $\mathrm{C}+$ trials (reinforced trials with the alternative auditory stimulus) were substituted for A+ trials in the training of the second. The third and fourth groups, respectively, were trained like the first two, except for the omission of the $\mathrm{B}$ stimulus. The procedure in the 3 test sessions (with B, A, and $\mathrm{C}$ ) was the same as in the first experiment.

In Experiment 3 (forward $\mathrm{C}+$ ), four groups of 14 subjects each were trained in two stages (see Table 1), with 15 sessions of $\mathrm{A}+$ or $\mathrm{C}+$ training in the first stage and 5 of $\mathrm{AB}+$ or $\mathrm{A}+$ training in the second, and with 10 trials in each of the sessions. This experiment was a forward version of Experiment 2, and thus it otherwise followed the same plan as that earlier experiment.

\section{RESULTS}

In Table 1, the results of Experiment 1 (concurrent KaminWagner) are summarized in terms of the mean number of responses made by each group in the nonreinforced test trials (i.e., the mean number of responses per testing session) with each of the three stimuli. The pattern of less responding to B, the target stimulus, in Group 1 than in Group 3 (the Wagner control) might be taken for a true blocking effect, were it not for the results for Group 2 (the Kamin control), which suggest that responding in the Wagner control group was elevated by the unsignaled contextual reinforcement. The same elevation is evident in the results for Group 6, although the level of responding to B in all of the B-absent groups was understandably less than in the B-present groups, for which B itself had been reinforced in training. The effect of unsignaled contextual reinforcement is evidenced also by intertrial (pre-CS) responding, which was somewhat greater in Groups 1 and 4 than in Groups 2 and 5 (owing presumably to the A+ trials), but substantially greater in Groups 3 and 6. The concern expressed by Blaser et al. (2004) in relation to their own use of Wagner's (unsignaled reinforcement) control - that lesser responding in the blocking group might be traced to enhanced contextual conditioning in the control group - is validated by these results.

An ANOVA based on responses to B by each of the six groups showed a significant effect of substitution (three levels: A+ trials vs. experience with the context alone instead of the $\mathrm{A}+$ trials vs. unsignaled reinforcements instead of the $\mathrm{A}+$ trials) $[F(2,42)=4.18, p<.05$; this level of confidence is the one adopted throughout], a significant $\mathrm{B}$ (presence vs. absence) effect $[F(1,42)=24.05]$, and a nonsignificant substitution $\times \mathrm{B}$ interaction $(F<1)$. A similar analysis of pre-CS responses showed a significant substitution effect $[F(2,42)=13.16]$ : The pooled Wagner control groups responded significantly more (according to Tukey HSDs) dur- ing the pre-CS period than did the pooled A+ groups or the pooled Kamin control groups, which did not differ. No other effects or interactions were significant $(F<1)$.

A separate analysis of responses to $B$ in the four groups of the Kamin experiment nested within Experiment 1 (Groups 1, 2,4 , and 5) showed a significant B effect $[F(1,28)=18.96]$, but neither a significant effect of substitution (A+ vs. context alone; $F<1)$ nor a significant substitution $\times \mathrm{B}$ interaction $[F(1,28)=1.24]$. A similar analysis for the four groups of the nested Wagner experiment (Groups 1,3,4, and 6) showed both a significant effect of substitution [A+ vs. unsignaled reinforcement; $F(1,28)=6.46]$ and a significant $\mathrm{B}$ effect $[F(1,28)=14.94]$, with no significant substitution $\times B$ interaction $[F(1,28)=1.49]$. The substitution effect in the Wagner experiment of course might be due to higher responding during the CS, induced by the unsignaled food during Phase 1 training in the control groups. An analysis of pre-CS responding indicated that this was indeed the case. For preCS responding in the Wagner experiment, there was a significant substitution effect $[F(1,28)=8.46]$, with no other significant effect on pre-CS responding. When we controlled for the level of pre-CS responding by using this variable as the covariate in an analysis of covariance, there was no longer a significant substitution effect $[F(1,27)=1.42]$, and only a significant effect of $\mathrm{B}$ remained $[F(1,27)=20.29]$.

The significant B effect in responding to B clearly indicates that a good deal was learned about it in the context of the $\mathrm{AB}$ compound, and a significant $\mathrm{B}$ effect in an analysis of responding to $\mathrm{A}$ by the six groups $[F(1,42)=8.81]$ suggests that A was to some extent overshadowed by $\mathrm{B}$. The level of responding in the test with $\mathrm{C}$, which the subjects had never previously encountered, was negligible. On the Hullian assumption that overshadowing can be understood in terms of afferent interaction, the entire pattern of results for the three stimuli follows readily from simple contiguity theory - that is, without the assumption that the stimuli competed for either associative strength or attention.

The results of Experiment 2 (concurrent $\mathrm{C}+$ ), summarized in Table 1, give no indication of blocking. An ANOVA based on responses to B showed a significant B effect $[F(1,47)=7.71]$, but neither a significant substitution effect $(\mathrm{A}+$ vs. $\mathrm{C}+; F<1)$ nor a significant substitution $\times \mathrm{B}$ interaction $(F<1)$. Pre-CS responding was negligible, and there were no significant differences between the groups in pre-CS responding. Nevertheless, we did an analysis of covariance on responding to $\mathrm{B}$ using pre-CS responding as the covariate. This analysis did not change the picture, yielding only a significant B effect $[F(1,41)=9.70]$. Again, the results are in accord with expectations from simple contiguity theory, as are those for tests with the other stimuli: That is, we found somewhat (although not significantly) more responding to $\mathrm{A}$ in the $\mathrm{A}+$ than in the $\mathrm{C}+$ groups $[F(1,42)=$ $2.89]$ and significantly more responding to $\mathrm{C}$ in the $\mathrm{C}+$ groups than in the $\mathrm{A}+$ groups $[F(1,42)=51.01]$.

Summarized also in Table 1 are the results for Experiment 3 (forward $\mathrm{C}+$ ). The responses to $\mathrm{B}$ here suggest a true blocking effect- a difference in the performance of the two conventional groups (1 and 2) that was not reflected in the performance of the two B-absent groups (3 and 4). An ANOVA showed a significant B effect $[F(1,52)=10.57]$ 
with no significant substitution effect $[F(1,52)=3.65]$, but there was a significant substitution $\times \mathrm{B}$ interaction $[F(1,52)=4.42]$. Pre-CS responding was again negligible, but to be sure that the results were not due to differences in pre-CS responding, we did an analysis of covariance with pre-CS responding as the covariate, which yielded the same pattern of results: that is, no significant effect of the covariate, but a significant $\mathrm{B}$ effect $[F(1,51)=10.55]$, no significant substitution effect $[F(1,51)=3.64]$, and a significant substitution $\times \mathrm{B}$ interaction $[F(1,5)=4.53]$. The difference in the performance of the two conventional groups was significant $[t(26)=2.06]$, but the difference between the two B-absent groups was not $[t(26)=0.48]$. As in Experiment 2 with the concurrent presentation of $\mathrm{C}+$, and again as would be expected from simple contiguity theory, there was somewhat (although not significantly) more responding to $\mathrm{A}$ in the $\mathrm{A}+$ than in the $\mathrm{C}+$ groups $[F(1,52)=$ 1.24] and significantly more responding to $\mathrm{C}$ in the $\mathrm{C}+$ than in the $\mathrm{A}+$ groups $[F(1,52)=34.31]$.

\section{DISCUSSION}

In the planning of these experiments, we confidently anticipated that the conventional groups would in each case give the traditional blocking result-or at least give the appearance of blocking - which the parallel B-absent treatment would help to evaluate. This proved true for the Wagner experiment embedded in Experiment 1 (concurrent Kamin-Wagner design), which seemed to show a concurrent blocking effect, but the difference in the test performance of the B-present groups, mirrored as it was in the performance of the B-absent groups, could readily be understood in terms of simple contiguity theory, on the assumption that responding on test trials with the target stimulus was enhanced by contextual reinforcement during training. The results of the Kamin experiment in Experiment 1 were negative, however, as were the results of Experiment 2 (concurrent $\mathrm{C}+$ ). These two failures to find what is usually conceived of as a rather robust effect might lead one to question the power of the technique employed. The entire pattern of results, however, suggests that substantial control of behavior actually was achieved. It may be reasonable to suspect that previous failures to find blocking, where it was expected, have on occasion not been reported because it was assumed that something was wrong with the experiments rather than with the expectations. The conditions necessary to give at least the appearance of blocking have not as yet been rigorously defined.

Evidence of a true blocking effect did, however, appear in Experiment 3 (forward $\mathrm{C}+$ ) - a difference in the test performance of the B-present groups that was not reflected in the performance of the B-absent groups. Experiment 3 closely resembled Experiment 2, except that the A+ or $\mathrm{C}+$ training preceded the $\mathrm{AB}+$ training rather than being concurrent with it. The absolute results of the two experiments also are very much alike, except that responding by Group 1 in the tests with a target stimulus was less in the forward than in the concurrent case. The greater effectiveness of the forward procedure was anticipated early on by Wagner (1969), on the assumption (which these data seem to support) that learning about $\mathrm{B}$ on any $\mathrm{AB}$ trial depends on what has already been learned about A.

It might be assumed on the basis of the results of Experiment 3 that positive results obtained in previous betweengroups experiments in which the $\mathrm{C}+$ control procedure was used can be taken at face value despite the lack of $\mathrm{B}$-absent treatments. It might be assumed also that, if the $\mathrm{C}+$ control procedure is used, B-absent treatments will be unnecessary in future experiments on blocking, about which, given the array of questionable control procedures used in the past, there is now very little dependable information. (The results of Experiment 1 certainly justify doubt as to the appropriateness of the Wagner control.) It might be well, however, to proceed more cautiously for a time, continuing in conventional between-groups experiments to rely on B-absent treatments despite the doubling of the number of experimental groups that is entailed. An attractive alternative, as Blaser et al. (2006) have suggested, is to turn to within-subjects designs (e.g., to test $\mathrm{B}$ vs. $\mathrm{D}$ after training with $\mathrm{A}+, \mathrm{AB}+$, and $\mathrm{CD}+$ ), in which a single group takes the place of four. Plausible evidence of blocking in rats has begun to be provided by such experiments as well (McNally \& Cole, 2006).

\section{AUTHOR NOTE}

This research was supported by NIH Grant R01MH068073 and NSF Grant IBN03-46546. We thank J. Chan, B. Mello, and M. Wan for assistance. Correspondence concerning this article should be addressed to M. E. Bitterman, Békésy Laboratory of Neurobiology, 1993 East-West Road, Honolulu, HI 96822 (e-mail: jeffb@pbrc.hawaii.edu).

\section{REFERENCES}

Blaser, R. E., Couvillon, P. A., \& Bitterman, M. E. (2004). Backward blocking in honeybees. Quarterly Journal of Experimental Psychology, 57B, 349-360.

Blaser, R. E., Couvillon, P. A., \& Bitterman, M. E. (2006). Blocking and pseudoblocking: New control experiments with honeybees. Quarterly Journal of Experimental Psychology, 59, 68-76.

Kamin, L. J. (1969). Selective association and conditioning. In N. J. Mackintosh \& W. K. Honig (Eds.), Fundamental issues in associative learning (pp. 42-64). Halifax, NS: Dalhousie University Press.

McNally, G. P., \& Cole, S. (2006). Opioid receptors in the midbrain periaqueductal gray regulate prediction errors during Pavlovian fear conditioning. Behavioral Neuroscience, 120, 313-323.

Miller, R. R., \& Matzel, L. D. (1988). The comparator hypothesis: A response rule for the expression of associations. In G. H. Bower (Ed.), The psychology of learning and motivation (Vol. 22, pp. 51-92). San Diego: Academic Press.

Rescorla, R. A. (1981). Within-signal learning in autoshaping. Animal Learning \& Behavior, 9, 245-252.

Rescorla, R. A., \& Wagner, A. R. (1972). A theory of Pavlovian conditioning: Variation in the effectiveness of reinforcement and nonreinforcement. In A. H. Black \& W. F. Prokasy (Eds.), Classical conditioning II: Current research and theory (pp. 64-99). New York: Appleton-Century-Crofts.

Smith, B. H., \& CoBEy, S. (1994). The olfactory memory of the honeybee, Apis mellifera: II. Blocking between odorants in binary mixtures. Journal of Experimental Biology, 195, 91-108.

Sutherland, N. S., \& Mackintosh, N. J. (1971). Mechanisms of animal discrimination learning. New York: Academic Press.

WAGNER, A. R. (1969). Stimulus validity and stimulus selection in associative learning. In N. J. Mackintosh \& W. K. Honig (Eds.), Fundamental issues in associative learning (pp. 90-122). Halifax, NS: Dalhousie University Press.

(Manuscript received November 12, 2007; revision accepted for publication December 12, 2007.) 